\title{
A GENERALIZATION OF THE FOURIER COSINE SERIES*
}

\author{
BY
}

\section{J. L. WALSH}

It is well known that on the interval $0 \leqq x \leqq \pi$ large classes of functions arbitrary except for certain restrictions as to oscillation or as to continuity and the existence and continuity of derivatives or as to some similar propertycan be developed into series in terms of the functions $\{\cos n x\}$. The series are of the type

$$
f(x)=\frac{a_{0}}{2}+a_{1} \cos x+a_{2} \cos 2 x+\cdots,
$$

where any coefficient $a_{n}$ may be obtained by the formal process of multiplying equation (1) through by $\cos n x d x$ and integrating term by term:

$$
a_{n}=\frac{2}{\pi} \int_{0}^{\pi} f(x) \cos n x d x .
$$

The present paper considers the problem of developing arbitrary functions throughout the same interval in terms of the second set of functions $\left\{\cos \lambda_{n} x\right\}$, where $\lambda_{n}$ is very near to $n$. Under suitable restrictions it is proved that the two developments have essentially the same convergence properties. $f$ We

* Presented to the Society, September, 1920.

† This study was undertaken at the suggestion of Professor Birkhoff, who proposed an analogous generalization of the Fourier sine series. There is no analogous generalization of the sine series such that developments in terms of the two sets of functions $\{\sin n x$ \} and $\left\{\sin \lambda_{n} x\right\}$, where $\lambda_{n}$ is near but not equal to $n$, will have the same convergence properties on the interval $0 \leqq x \leqq \pi$. For at the right-hand end of the interval any particular function $\sin \lambda_{k} x$ is not zero and hence cannot, be developed in a uniformly convergent series in terms of \{sin $n x\}$, but it can be developed in a uniformly convergent series in terms of $\left\{\sin \lambda_{n} x\right.$ \}. Thus the methods of the present paper cannot be used to give a generalization of the sine series on the interval $0 \leqq x \leqq x$, but these methods with slight modifications would enable us to replace the set $\left\{\sin n x\right.$ \} by a set $\left\{\sin \lambda_{n} x\right.$ \} such that developments in terms of the two sets of functions will have the same convergence properties on the interval $0 \leqq x \leqq x-\epsilon$, where - is arbitrary.

The methods used in this paper are in spirit closely related to the methods used by Birkhoff, Paris Comptes Rendus, vol. 164 (1917), pp. 942-945, who gives a similar generalization of Taylor's series. Birkhoff makes use of the theory of integral equations, but the related theory of infinitely many variables as used in the present paper leads to more general results. 
shall say that two series

$$
\sum_{i=1}^{\infty} a_{i} \bar{u}_{i}, \quad \sum_{i=j}^{\infty} b_{i} u_{i}
$$

have essentially the same convergence properties when and only when the series

$$
\sum_{i=1}^{\infty}\left(a_{i} \bar{u}_{i}-b_{i} u_{i}\right)
$$

converges absolutely and uniformly to the sum zero.

The set of functions

$$
\frac{1}{\pi}, \frac{2}{\pi} \cos x, \frac{2}{\pi} \cos 2 x, \cdots
$$

is a normal and orthogonal set on the interval $0 \leqq x \leqq \pi$. Any set of functions $\left\{\bar{u}_{n}\right\}$ continuous on a finite interval is said to be normal and orthogonal on that interval if and only if

$$
\int \bar{u}_{i} \bar{u}_{j} d x=\delta_{i j} \quad(i, j=1,2,3, \cdots)
$$

here and throughout this paper $\delta_{i j}$ is the Kronecker symbol which is unity or zero according as $i$ and $j$ are or are not equal. In this integral and below we omit the argument, which is $x$ in every case, and also omit the limits of integration, which are the ends of the interval considered. We shall prove that corresponding to the set of functions

$$
\frac{1}{\pi} \cos \lambda_{0} x, \frac{2}{\pi} \cos \lambda_{1} x, \frac{2}{\pi} \cos \lambda_{2} x, \cdots
$$

there is another set of functions such that the two sets are biorthogonal for $0 \leqq x \leqq \pi$. Two sets of continuous functions $\left\{u_{n}\right\}$ and $\left\{u_{n}\right\}$ are biorthogonal on an interval if and only if

$$
\int u_{i} v_{j} d x=\delta_{i j} \quad(i, j=1,2, \cdots) .
$$

We shall prove a general theorem concerning the generalization of expansions in terms of any uniformly bounded normal orthogonal set $\left\{\bar{u}_{n}\right\}$, by considering a set $\left\{u_{n}\right\}$ where $u_{n}$ is a function neighboring to $\bar{u}_{n}$. Briefly described, the method is to expand the $u_{n}$ into series in terms of the $\bar{u}_{n}$, to invert the corresponding system of equations and expand the $\bar{u}_{n}$ in terms of the $u_{n}$, and finall! to substitute the latter series into the expansion of the arbitrary function in terms of the $\bar{u}_{n}$. This gives the expansion of $f(x)$ in terms of the $u_{n}$. The form of presentation of the material is slightly changed by the introduction of a set of functions $\left\{v_{n}\right\}$ such that $\left\{u_{n}\right\}$ and $\left\{v_{n}\right\}$ are biorthogonal sets. The rarious steps in the proof will seem much less artificial if the general nethod is kept in mind. 
After the general theorem is proved, application is made to the cosine series. We add that the method seems to be of very wide applicability; it is hoped later to give further applications to the Sturm-Liouville and other series developments. Throughout the present paper there is more regard for simplicity than for ultimate generality.

\section{A genERAL THEOREM}

Theorem I: Suppose that $\left\{\bar{u}_{n}\right\}$ is a set of uniformly bounded normal orthogonal functions in an interval, and that in this interval $\left\{u_{n}\right\}$ is a set of uniformly bounded continuous functions each of which can be developed into a series

$$
u_{n}=\sum_{k=1}^{\infty}\left(c_{n k}+\delta_{n k}\right) \bar{u}_{k} \quad(n=1,2, \cdots),
$$

where the coefficients have the values

$$
c_{n k}+\delta_{n k}=\int u_{n} \bar{u}_{k} d x .
$$

Suppose further that the three series

$$
\sum_{k, k=1}^{\infty} c_{i k}^{2}, \sum_{i=1}^{\infty}\left(\sum_{k=1}^{\infty} c_{i k}^{2}\right)^{i}, \sum_{k=1}^{\infty}\left(\sum_{i=1}^{\infty} c_{i k}^{i}\right)^{k}
$$

converge and that the value of the first is less than unity.

Then there exists a set of functions $\left\{v_{i}\right\}$ such that $\left\{u_{i}\right\}$ and $\left\{v_{i}\right\}$ are biorthogonal sets:

$$
\int u_{i} v_{j} d x=\delta_{i j} \quad(i, j=1,2, \cdots) .
$$

Furthermore, if $f(x)$ is any function integrable and with an integrable square (in the sense of Lebesgue), then the two series

$$
\begin{aligned}
& f(x) \sim \sum_{i=1}^{\infty} a_{i} \bar{u}_{i}, \\
& f(x) \sim \sum_{i=1}^{\infty} b_{i} u_{i},
\end{aligned}
$$

onhere

$$
\begin{aligned}
& a_{i}=\int f \bar{u}_{i} d x, \\
& b_{i}=\int f v_{i} d x,
\end{aligned}
$$

have essentially the same convergence properties.

The sign $\sim$ is used simply to indicate that the coefficients $a_{i}$ and $b_{i}$ are given 
by (8). We shall give later some of the more immediate consequences of the fact that (6) and (7) have essentially the same convergence properties.

To prepare for the inversion of system (2), which is an important step in the proof of Theorem I, we shall use the following

LEMMA: If for the system

$$
\begin{array}{r}
\left(1+c_{11}\right) x_{1}+\quad c_{12} x_{2}+\quad c_{13} x_{3}+\cdots=c_{1}, \\
c_{21} x_{1}+\left(1+c_{22}\right) x_{2}+\quad c_{23} x_{8}+\cdots=c_{2}, \\
c_{31} x_{1}+\quad c_{32} x_{2}+\left(1+c_{33}\right) x_{3}+\cdots=c_{3},
\end{array}
$$

the series

$$
\gamma^{2}=\sum_{j=1}^{\infty} c_{j}^{2}, \quad p^{2}=\sum_{i, j=1}^{\infty} c_{i j}^{2}
$$

converge, and if $p^{2}<1$, then the system has one solution $\left\{x_{i}\right\}$ and only one solution for which

$$
\sum_{i=1}^{\infty} x_{i}^{2}
$$

converges.*

The lemma is proved by the method of successive approximations, setting

$$
\begin{aligned}
x_{i}^{(1)} & =c_{i}, \\
x_{i}^{(2)} & =c_{i}-\left[c_{i 1} x_{1}^{(1)}+c_{i 2} x_{2}^{(1)}+\cdots\right], \\
\cdot & \cdot \cdot \cdot \cdot \cdot \cdot \cdot \cdot \cdot \cdot \cdot \\
x_{i}^{(n+1)} & =c_{i}-\left[c_{i 1} x_{1}^{(n)}+c_{i 2} x_{2}^{(n)}+\cdots\right],
\end{aligned}
$$

From the well-known inequality

$$
\sum_{k=1}^{n}\left|\alpha_{k} \beta_{k}\right| \leqq\left(\sum_{k=1}^{n} \alpha_{k}^{2}\right)^{1}\left(\sum_{k=1}^{n} \beta_{k}^{2}\right)^{\frac{1}{2}}
$$

(which holds for all sets of numbers $\left\{\alpha_{k}\right\}$ and $\left\{\beta_{k}\right\}$ ) and from the convergence of the series whose sums are denoted by $\gamma^{2}$ and $p^{2}$, it follows that the series

$$
x_{i}=x_{i}^{(1)}+\left(x_{i}^{(2)}-x_{i}^{(1)}\right)+\left(x_{i}^{(3)}-x_{i}^{(2)}\right)+\cdots \quad(i=1,2, \cdots)
$$

are all absolutely convergent and that the system $\left\{x_{i}\right\}$ as thus defined is a

* This lemma is included implicitly in some results of Hilb, Sitzungsberichte der Physikalisch-medizinischen Sozietat in Erlangen, vol. 40 (1908), pp. 1-6. Hilb makes use of the theory of bilinear forms in infinitely many variables, so that his proof differs in form (but not in substance) from the proof here presented. We indicate the proof because of its simplicity and because we shall use later the inequalities obtained in the course of the proof. 
solution of (9). If we introduce the notation $p_{i}^{2}=\sum_{j=1}^{\infty} c_{i j}^{2}$, it is readily proved by further use of the same inequality that

$$
\begin{gathered}
\left|x_{i}-c_{i}\right| \leqq \frac{\gamma p_{i}}{1-p}, \\
\sum_{i=1}^{\infty}\left|x_{i}-c_{i}\right|^{2} \leqq \frac{\gamma^{2} p^{2}}{(1-p)^{2}} .
\end{gathered}
$$

The series $\sum_{i=1}^{\infty} x_{i}^{2}$ converges, for we obtain the relations*

$$
\begin{gathered}
\left|x_{i}\right| \leqq\left|x_{i}-c_{i}\right|+\left|c_{i}\right|, \\
\sum_{i=1}^{\infty} x_{i}^{2} \leqq \sum_{i=1}^{\infty}\left(\left|x_{i}-c_{i}\right|+\left|c_{i}\right|\right)^{2} \leqq 2 \sum_{i=1}^{\infty}\left(x_{i}-c_{i}\right)^{2}+2 \sum_{i=1}^{\infty} c_{i}^{2} .
\end{gathered}
$$

There is no other solution of system (9) such that $\sum_{i=1}^{\infty} x_{i}^{2}$ converges. For if there were, we should have a solution $\left\{y_{i}\right\}$ of the homogeneous sirstem corresponding to (9) and such that the series.

$$
Y^{2}=\sum_{i=1}^{\infty} y_{i}^{2}
$$

converges. In the $k$ th equation of the homogeneous system, if we transpose $y_{k}$ to the right-hand member, we obtain

and hence we also have

$$
y_{k}^{2} \leqq Y^{2} p_{k}^{2},
$$

$$
\sum_{k=1}^{\infty} y_{k}^{2} \leqq Y^{2} p^{2}
$$

In this same manner we have

$$
\sum_{k=1}^{\infty} y_{k}^{2} \leqq Y^{2} p^{2 n},
$$

where $n$ is any positive integer, and hence $y_{k}=0(k=1,2, \cdots)$.

We apply this lemma (the proof of which is now complete) to determine the set of functions $\left\{v_{k}\right\}$ of Theorem I. Let us assume a development of the type

$$
v_{k}=\sum_{i=1}^{\infty}\left(d_{k i}+\delta_{k i}\right) \bar{u}_{i}, \quad \text { where } \quad d_{k i}+\delta_{k i}=\int v_{k} \bar{u}_{i} d x .
$$

The condition that the $x_{k}$ have the desired property (5) is given formally from the developments (2) and (12) by the conditions

$$
c_{k i}+d_{i k}+c_{k 1} d_{i 1}+c_{k 2} d_{i 2}+c_{k 3} d_{i 3}+\cdots=0
$$

for every $i$ and $k$. When $i$ is kept fixed, this is preciselv system (9): the

* By use of the inequality

$$
(\alpha+\beta)^{2} \leqq 2\left(\alpha^{2}+\beta^{2}\right)
$$


conditions of the lemma are fulfilled by rirtue of the hrpothesis of Theorem I, and therefore there exists a solution $\left(d_{i 1}, d_{i 2}, d_{i 3}, \cdots\right)$, with the properties stated in the lemma. If we choose that solution and use inequalities (11) and the convergence of series (4), we actually arrive at a șistem of continuous functions $\left\{c_{k}\right\}$ which satisfy (j) and are given by (12) and (13).

Our preparation for the inversion of system (2) is nearly complete. Te introduce the notation

$$
\epsilon_{i k}=c_{k i}+d_{i k}+c_{1 i} d_{1 k}+c_{2 i} d_{2 k}+c_{3 i} d_{3 k}+\cdots
$$

and shall prove $\epsilon_{i k}=0$ for every $i$ and $k$. In the set of equations

$$
\begin{aligned}
& \epsilon_{i 1}=c_{1 i}+d_{i 1}+c_{1 i} d_{11}+c_{2 i} d_{21}+c_{3 i} d_{31}+\cdots, \\
& \epsilon_{i 2}=c_{2 i}+d_{i 2}+c_{1 i} d_{12}+c_{2 i} d_{22}+c_{3 i} d_{32}+\cdots, \\
& \epsilon_{i 3}=c_{3 i}+d_{i 3}+c_{1 i} d_{13}+c_{2 i} d_{23}+c_{3 i} d_{33}+\cdots,
\end{aligned}
$$

multiply the ith equation by $c_{n i}$ and sum by columns, making use of (13) and (1t). We obtain the result

$$
c_{k 1}^{2} \epsilon_{i 1}+c_{k 2} \epsilon_{i 2}+c_{k 3} \epsilon_{i 3}+\cdots=-\epsilon_{i k} .
$$

The formal work is readily justified by the convergence of series (4). Moreover, the series $\sum_{k=1}^{\infty} \epsilon_{i k}^{2}$ converges, from the definition (14) and from (11) applied to the set $\left(d_{i 1}, d_{i 2}, d_{i 3}, \cdots\right)$ as a solution of $(13)$, so the set $\left(\epsilon_{i 1}, \epsilon_{i 2}\right.$, $\left.\epsilon_{i 3}, \cdots\right)$ forms a solution of the homogeneous svistem corresponding to (9) and hence $\epsilon_{i k}=0$ for every $i$ and $k$.

We are now in a position to invert system (2). We write the equations

$$
\begin{aligned}
& c_{k 1}+d_{1 k}+c_{11} d_{1 k}+c_{21} d_{2 k}+c_{31} d_{3 k}+\cdots=0, \\
& c_{k 2 .}+d_{2 k}+c_{12} d_{1 k}+c_{22} d_{2 k}+c_{32} d_{3 k}+\cdots=0, \\
& c_{k 3}+d_{3 k}+c_{13} d_{1 k}+c_{23} d_{2 k}+c_{33} d_{3 k}+\cdots=0,
\end{aligned}
$$

multiply the ith equation by $\bar{u}_{i}$ and sum by columns. Justification of the formal work is immediate, and we obtain the desired derelopments

$$
\bar{u}_{k}-u_{k}=\sum_{i=1}^{\infty} d_{i k} u_{i} \quad(k=1,2,3, \cdots) .
$$

Let us suppose, now, $f^{\prime}(x)$ to be any function integrable and with an integrable square in the sense of Lebesgue. Te find the coefficients (S) by multi-

* For a non-ranishing finite determinant, the numbers $d_{i k}+\delta_{i k}$ are the quotients of the cofactors of the $c_{i k}+\delta_{i k}$ by the value of the determinant. 
plying (12) through by $f(x)$ and integrating term by term. This yields the result

$$
b_{k}-a_{k}=d_{k 1} a_{1}+d_{k 2} a_{2}+d_{k 3} a_{3}+\cdots .^{*}
$$

The difference of the series (6) and (7) may be written

$$
\begin{aligned}
& \sum_{1=i}^{\infty}\left(a_{i} \bar{u}_{i}-b_{i} u_{i}\right)=\sum_{i=1}^{\infty} a_{i}\left(\bar{u}_{i}-u_{i}\right)+\sum_{i=1}^{\infty}\left(a_{i}-b_{i}\right) u_{i} \\
& =-\sum_{i=1}^{\infty} a_{i}\left(\sum_{j=1}^{\infty} c_{i j} \bar{u}_{j}\right)-\sum_{i=1}^{\infty}\left(d_{i 1} a_{1}+d_{i 2} a_{2}+\cdots\right)\left(\bar{u}_{i}+\sum_{j=1}^{\infty} c_{i j} \bar{u}_{j}\right),
\end{aligned}
$$

which series converges absolutely and uniformly and has the sum zero. We make use of the convergence of the series $\sum_{i=1}^{\infty} a_{i}^{2}$, which may be proved easily from the relation

$$
\int\left(f-a_{1} \bar{u}_{1}-a_{2} \bar{u}_{2}-\cdots-a_{n} \bar{u}_{n}\right)^{2} d x \geqq 0 .
$$

Hence, series (6) and (7) have the same convergence properties, in the sense that (16) converges absolutely and uniformly to zero. It follows immediately that properties of absolute convergence, convergence, summability, and divergence obtain for one series as for the other, and likewise the properties of uniform convergence in the entire interval considered or in any sub-interval, uniform summability, and also of term-by-term integrability. Whenever the two series (6) and (7) are convergent, summable, or properly divergent, their sums are the same. The nature of the approximating functions and of their approach to the limit (in case of convergence) at a point of continuity or of discontinuity of $f(x)$ is essentially the same for (6) as for (7). In particular if Gibbs's phenomenon occurs for (6) it also occurs for (7). The reader will notice various other properties common to the sets $\left\{\bar{u}_{i}\right\}$ and $\left\{u_{i}\right\}$, such as the existence or non-existence of a continuous function for which the formal series does not converge at every point.

The conditions of Theorem I concerned with the series (4) can be made

* If we use the convergence of $\Sigma_{i=1}^{\infty} a_{i}^{2}$, which is proved from (17); if we write the equations

$$
\begin{aligned}
& b_{1}-a_{1}=d_{11} a_{1}+d_{12} a_{2}+d_{13} a_{3}+\cdots, \\
& b_{2}-a_{2}=d_{21} a_{1}+d_{22} a_{2}+d_{23} a_{3}+\cdots, \\
& b_{3}-a_{3}=d_{31} a_{1}+d_{32} a_{2}+d_{33} a_{3}+\cdots,
\end{aligned}
$$

multiply the $i$ th equation by $c_{i k}$, and sum by columns, we obtain

$$
a_{k}-b_{k}=c_{1 k} b_{1}+c_{2 k} b_{2}+c_{3 k} b_{3}+\cdots \text {. }
$$

Hence if the $\left\{b_{i}\right\}$ are all zero, so are all the $\left\{a_{i}\right\}$. The converse is evident from (15).

We also add that if the series $\Sigma_{i=1}^{\infty}\left|a_{i}\right|$ converges, so does the series $\Sigma_{i=1}^{\infty}\left|b_{i}\right|$, and conversely. 
more restrictive so that the convergence properties of (6) and (7) will be essentially the same for all functions absolutely integrable in the sense of Lebesque, or on the other hand can be made less restrictive and still vield a result say for functions such that the series of absolute values of the coefficients $\sum_{i=1}^{\infty}\left|a_{i}\right|$ converges.

We add the remark that the relation between the $\left\{u_{i}\right\}$ and the $\left\{v_{i}\right\}$ is essentially reciprocal-we may obtain corresponding results for expansions in terms of the $\left\{v_{i}\right\}$. Theorem I is readily extended, moreover, by taking as point of departure developments in terms of functions $\left\{\bar{u}_{i}\right\}$ which are not supposed orthogonal.

\section{Applichtion to the Fourier cosine series}

For the interval $0 \leqq x \leqq \pi$, we have mentioned the normal orthogonal system of uniformly bounded continuous functions

$$
\frac{1}{\pi}, \frac{2}{\pi} \cos x, \frac{2}{\pi} \cos 2 x, \frac{2}{\pi} \cos 3 x, \cdots .
$$

Application of Theorem I will yield the following theorem.

Theorem II. The expansion of any function $f(x)$ integrable and with an integrable square in terms of the functions

$$
\frac{1}{\pi} \cos \lambda_{0} x, \frac{2}{\pi} \cos \lambda_{1} x, \frac{2}{\pi} \cos \lambda_{2} x, \frac{2}{\pi} \cos \lambda_{3} x, \cdots
$$

on the interval $0 \leqq x \leqq \pi$, where

$$
\lambda_{0}^{2}+4\left(\lambda_{1}-1\right)^{2}+4\left(\lambda_{2}-2\right)^{2}+4\left(\lambda_{3}-3\right)^{2}+\cdots<\frac{1}{\pi}
$$

and where

$$
\sum_{n=1}^{\infty} n^{2}\left|\lambda_{n}-n\right|
$$

converges, has essentially the same convergence properties as the expansion of $f(x)$ in terms of the functions (18).

It is of course true that our set of functions $\left\{u_{m}\right\}$ can be developed in terms of the functions $\left\{\bar{u}_{m}\right\}$, so it merely remains to prove the convergence of the series (4) and that the value of the first of these series is less than unity.

Inequality (17) gives us the relation

$$
\sum_{k=1}^{\infty} c_{m k k}^{2} \leqq \int\left(\bar{u}_{m}-u_{m}\right)^{2} d x \quad(m=1,2,3, \cdots),
$$

and in the present case if $m \neq 1$ and if we replace $m$ by $n+1$, this integral 
becomes

$$
\begin{aligned}
\frac{4}{\pi^{2}} \int_{0}^{\pi}\left(\cos \lambda_{n} x-\right. & \cos n x)^{2} d x \\
& =\frac{4}{\pi^{2}} \int_{0}^{\pi}\left[4 \sin ^{2} \frac{1}{2}\left(\lambda_{n}+n\right) x \cdot \sin ^{2} \frac{1}{2}\left(\lambda_{n}-n\right) x\right] d x .
\end{aligned}
$$

But we always have the relation $|\sin \alpha| \leqq|\alpha|$, and hence we obtain

$$
\sum_{k=1}^{\infty} c_{n+1, k}^{2} \leqq 4 \pi\left(\lambda_{n}-n\right)^{2} \quad(n=1,2,3, \cdots)
$$

If we omit the factor 4 from the right-hand member we obtain the propes formula for $n=0$. Satisfaction of (19) therefore means that we have satis. fied the foliowing requirement of Theorem I

$$
\sum_{m, k=1}^{\infty} c_{m k}^{2}<1
$$

It remains to be shown that the last two series in (4) converge. From the formula

$$
c_{n+1, k+1}=\frac{4}{\pi^{2}} \int_{0}^{\pi}\left(\cos \lambda_{n} x-\cos n x\right) \cos k x d x
$$

$$
(n=1,2, \cdots ; k=1,2, \cdots)
$$

integration by parts twice yields the formula

$$
\begin{aligned}
\frac{\pi^{2}}{4} c_{n+1, k+1}=-\left[\left(\lambda_{n} \sin \lambda_{n} \cdot x\right.\right. & \left.-n \sin n x) \frac{\cos k x}{k^{2}}\right]_{0}^{\pi} \\
& +\int_{0}^{\pi} \cdot\left(\lambda_{n}^{2} \cos \lambda_{n} x-n^{2} \cos n x\right) \frac{\cos k \cdot x}{k^{2}} d x .
\end{aligned}
$$

To deal with this integral, we write

$$
\lambda_{n}^{2} \cos \lambda_{n} x-n^{2} \cos n x=\left(\lambda_{n}^{2}-n^{2}\right) \cos \lambda_{n} x+n^{2}\left(\cos \lambda_{n} x-\cos n x\right) .
$$

If $n>2$, we find with the aid of (19) the obvious relation

whence

$$
\left|\lambda_{n}+n\right|<2 n+2<n^{2}
$$

$$
\left|\lambda_{n}^{2}-n^{2}\right|<n^{2}\left|\lambda_{n}-n\right| \text {; }
$$

we use this inequality in considering the first term of the right-hand member of (24). The last term of the right-hand member of (24) can be transformed as in (21), and its absolute value is therefore not greater than $n^{2} \pi\left|\lambda_{n}-n\right|$.

The first term in the right-hand member of (23) with the factor $\cos k \pi / k^{2}$ omitted, reduces to $\lambda_{n}\left(\sin \lambda_{n} \pi-\sin n \pi\right)$. When $n>1$, we have $\left|\lambda_{n}\right| \leqq n^{2}$, and therefore

$$
\left|\lambda_{n}\left(\sin \lambda_{n} \pi-\sin n \pi\right)\right| \leqq n^{2} \pi\left|\lambda_{n}-n\right| .
$$


From these inequalities for the absolute value of the right-hand nituber of (23) and from the corresponding results for $k=0$ and $n \leqq 2$ we prove the convergence of

$$
\sum_{n, k=1}^{\infty}\left|c_{n k}\right|
$$

by means of the convergence of $(20)$ and hence* prove the convergence of the last two series of (4) and complete the proof of Theorem II.

We add the remark that a closer evaluation of $c_{n k}$, and also the consideration of functions $u_{n}=\mu_{n} \cos \lambda_{n} x$ where $\mu_{n}$ is properly determined, will give broader restrictions than (19) and (20).

HARVARD UNIVERSITY, May, 1920

* Here we make use of the inequality

$$
\sum_{i=1}^{n}\left|\alpha_{i}\right|=\left[\left(\sum_{i=1}^{n}\left|\alpha_{i}\right|\right)^{2}\right]^{k} \geqq\left[\sum_{i=1}^{n} \alpha_{i}^{2}\right]^{i} .
$$

